\title{
Fatores determinantes para a elaboração de estratégias com vistas à aceitação e uso da Realidade Aumentada em cenários de negócio*
}

João Vitor de Matos Silva' Ildeberto Aparecido Rodello ${ }^{2}$
* Recebido em: 22/06/2016. Aprovado em: 23/03/2017.

1 Professor da Universidade de São Paulo (USP) - Faculdade de Economia, Administração e Contabilidade de Ribeirão Preto (FEARP) - Departamento de Administração.

2 Professor da Universidade de São Paulo (USP) - Faculdade de Economia, Administração e Contabilidade de Ribeirão Preto (FEARP) - Departamento de Administração.

\section{A study about the acceptance and use of Augmented Reality applied to business scenarios according to UTAUT theory}

\section{Resumo}

Apesar de crescente, a utilização da Realidade Aumentada, ainda, requer estudos para confirmar seus benefícios e dificuldades, principalmente para a elaboração de estratégias quando aplicada a cenários de negócios. Nesse sentido, este artigo apresenta um estudo baseado na aplicação da Teoria Unificada de Aceitação e Uso da Tecnologia para identificar os principais itens relacionados com a aceitação e o uso da Realidade Aumentada no contexto do Marketing. A pesquisa envolveu a aplicação de um questionário adaptado da Teoria Unificada para 88 participantes. Na análise dos dados, foram utilizadas técnicas multivariadas de análise fatorial, teste de Scree e rotação do tipo Varimax para interpretação dos resultados. As respostas apontaram que os itens fundamentais para a aceitação e uso da Realidade Aumentada foram a Intenção Comportamental e a Expectativa de Esforço. O trabalho tem como limitações os poucos estudos na área da Realidade Aumentada em cenários de negócios, o que, ao mesmo tempo, ressalta sua singularidade e, também, o escopo da amostra alcançada. Porém, apesar das limitações, este artigo tem importância por analisar uma tecnologia emergente e promissora para cenários de negócios, e contribui, a partir dos resultados encontrados, com o destaque de potenciais fatores para que as empresas alcancem sucesso no desenvolvimento de ações baseadas em Realidade Aumentada.

Palavras-chave: UTAUT. Realidade Virtual e Aumentada. Tecnologia da Informação.

\section{Abstract}

Despite increasing, the Augmented Reality usage still requires studies to confirm their benefits and difficulties, mainly for the development of strategies to business scenarios application. Concerning this focus, this paper presents a study based on the application of the Unified Theory of Acceptance and Use of Technology to identify the main items related to the acceptance and use of Augmented Reality in the marketing scenarios. The research involved the application of an adapted survey from the Unified Theory to 88 participants. In the data analysis were used multivariate techniques of factor analysis, Scree test and rotation of Varimax type to interpret the results. The answers showed that the key items for the acceptance and use of Augmented Reality were the Behavioral Intention and the Effort Expectancy. This paper has as limitation the few studies in the Augmented 
Reality applied to business scenarios, which at the same time emphasizes their uniqueness and scope of the sample reached. However, despite the limitations, it is important for analyzing an emerging and promising technology to business scenarios, and contributes, from the results, with the highlight of potential factors for companies to achieve success in the development of actions based on Augmented Reality.

Keywords: UTAUT. Virtual and Augmented Reality. Information Technology.

\section{Introdução}

A Realidade Aumentada (RA) é uma área relacionada com evolução da interface humano-computador (IHC), visando tornar mais intuitiva as interações do usuário com as aplicações computacionais (KIRNER; SISCOUTTO, 2007). Azuma et al. (1997) (2001) definem RA como um sistema que permite ao usuário ver o mundo real com objetos virtuais sobrepostos ou compostos. Esse sistema tem as seguintes três características: combina o real e o virtual, é interativo em tempo real e está registrado em três dimensões.

Esse conceito de RA surgiu com o avanço das pesquisas sobre a Realidade Virtual (RV), que teve suas origens nos anos 60, principalmente pelas pesquisas de Sutherland (1968). No entanto, a tecnologia da época, ainda, não fornecia condições para a sua execução e evolução. Logo, somente na década de 90, com o desenvolvimento tecnológico, foi possível propiciar condições para a sua execução. Segundo Kirner e Siscoutto (2007), "apesar das potenciais vantagens da RV, a tecnologia demandava equipamentos especiais para que o usuário pudesse realizar suas interações no espaço da aplicação, e também causava um desconforto e dificuldades para o usuário na interação”. Esse é um exemplo do fatores barraram a uma maior utilização e disseminação da RV na época.

Se, por um lado, a RV encontrava barreiras para a sua utilização, por outro, a evolução tecnológica propiciou, na década de 90, o crescimento da RA, permitindo a sobreposição de objetos, informações e ambientes virtuais com o ambiente físico, por meio de algum dispositivo tecnológico (KIRNER; SISCOUTTO, 2007). Segundo White et al. (2014), o termo RA, isoladamente, é uma abordagem usada, especificamente, para visualizar informações digitais sobrepostas em imagens físicas e poder manipular tais informações por meio de interações com os objetos do mundo real. Em sistemas de RA, os sensores (como a câmera de um dispositivo móvel) são usados para derivar informações sobre as interações do usuário com o mundo real.

Segundo Van Kleef et al. (2010), "Apesar da grande quantidade de possibilidades com aplicações de RA, apenas alguns modelos de negócios exploram o potencial da tecnologia de RA. Isso pode ser causado por não ser claro para as empresas como elas podem agregar valor com a RA".

Segundo Rodello e Brega (2011), "Utilizando a RA a empresa é capaz de utilizar os quatro elementos do paradigma de novas comunicações entreter, informar, persuadir e interagir". Segundo a revista EXAME (2013), algumas empresas como Ray Ban, Lacta, Stella Artois, Volvo, dentre outras já utilizam a tecnologia de RA como ferramenta de Marketing, fornecendo experiências únicas aos seus consumidores. Porém, ainda segundo Rodello e Brega (2011), a ferramenta, ainda, tem custo elevado e o seu funcionamento é melhor observado com produtos que requerem uma grande interação do usuário.

Para indicar estratégias para utilização mais assertiva da RA em cenários de negócio, realizou-se este estudo sobre a aceitação do usuário (consumidor) em situações de uso da RA no Marketing de produtos. O modelo utilizado para essa finalidade foi o proposto por Venkatesh et al. (2003), UTAUT (Teoria Unificada de Aceitação e Uso da Tecnologia), que, atualmente, é considerado o modelo mais completo, uma vez que abrange 8 outros estudos de aceitação de tecnologia e os unifica em um modelo unificado.

Com a análise da aceitação e uso da RA, procurou-se destacar fatores que influenciam os usuários e são determinantes na sua aceitação, bem como no seu uso, contribuindo para o auxílio a gestores e empresas na elaboração de estratégias e de ferramentas que utilizam a RA, podendo potencializar a probabilidade de agregação valor para os seus negócios.

\section{Realidade Virtual e Aumentada aplicada a negócios}

Segundo Morie (1994), "A RV consiste em um ambiente tridimensional totalmente gerado por computador, denominado Ambiente Virtual (AV) dentro do qual o usuário pode agir de forma intuitiva e idêntica ao seu cotidiano". 
Inicialmente, a RA teve uma abordagem um pouco mais restrita, ao ser definida como uma forma de $\mathrm{RV}$, em que um visor montado na cabeça do usuário é transparente, permitindo uma visão clara do mundo real. Posteriormente, Milgram e Kishino (1994) propuseram o continuum de virtualidade para tentar distinguir melhor os conceitos (Figura 1).

Figura 1 - Representação simplificada do continuum de virtualidade

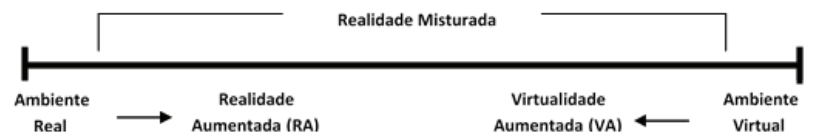

Fonte: Milgram e Kishino, 1994.

O continuum virtualidade pode ser usado para descrever um conceito que na verdade não existe em uma escala contínua que varia entre uma abordagem completamente virtual e o totalmente real. Por isso, engloba todas as variações possíveis e composições de objetos reais e virtuais.

A área entre os dois extremos, em que tanto o real e virtual são misturados, é denominada Realidade Misturada (Mixed Reality). Esta, por sua vez, consiste de duas variações: Realidade Aumentada (RA), onde o virtual aumenta o real, e Virtualidade Aumentada (VA), em que o real aumenta o virtual. Nesse ponto, RA pode ser pensada como o "meio termo" entre o ambiente virtual (completamente sintético) e telepresença (completamente real) (MILGRAM; KISHINO, 1994) (MILGRAM et al., 1994).

Segundo Kleef et al. (2010), a RA tem sido uma área de pesquisa ao longo das décadas, porém, em se tratando da área de negócios que essa tecnologia poderia impactar, existem poucos estudos. Segundo Rodello et. al. (2010), como áreas onde a RV e a RA estão sendo aplicadas, é possível citar área de Educação por conta da sua interação intuitiva, a área de entretenimento com jogos e filmes, a indústria de construção civil e, também, a área da saúde. Ainda segundo Kleef et al. (2010), apesar de uma grande quantidade de aplicações para a RA, poucos modelos de negócios a exploram efetivamente. Isso pode ser causado pelo fato de as empresas não saberem como utilizar a RA, o que está relacionado, diretamente, com a falta de estudos na área.

A utilização da RA pode trazer diversos benefícios para as organizações, como: alavancar vendas de produtos por meio de uma interação do cliente durante o processo de compra do produto, com o uso de propagandas para smartphones, computadores e televisões utilizando a RA, modelagem de roupas para os clientes a distância. Isso pode funcionar como uma forma de inovação das suas estratégias de Marketing, de relacionamento com o cliente, entre outros. Atualmente, é possível encontrar a RA sendo utilizada por museus em aplicativos para smartphones, que é o caso do Chess. Segundo Grandelle e Jansen (2015), o Chess consiste num aplicativo para smartphones e tablets que oferece a curadores ferramentas e recursos interativos de RA para personalizar as experiências dos visitantes de seus museus.

Outros exemplos da utilização da RA na área de negócios são, por exemplo, na engenharia civil, em que, segundo Cardozo (2015), é possível fazer uso dessa tecnologia em quase todas as etapas de um projeto e até mesmo como forma educativa nas universidades. Na etapa de elaboração de um projeto, é possível verificá-lo no ambiente real para averiguar alguns aspectos de dimensões e compatibilidade. Ainda segundo Cardozo (2015), a RA é um dos recursos tecnológicos a oferecer uma gama de aplicações possíveis em processos existentes em Arquitetura e Construção.

A RA, também, é uma alternativa para treinamento de pessoal em empresas, uma vez que dá vida ao conhecimento, transformando o aprendizado em algo mais marcante e facilitando a fixação de conteúdo. Ao tornar a situação algo real, possibilitando a imersão e a capacidade de interagir, o colaborador retém melhor a informação e passa a ter um entendimento global da empresa. (CASTANHA, 2015)

Segundo Bellini et. al (2016), a RV e a RA tem o potencial de se tornarem uma indústria multibilionária, e a previsão é de que essa indústria movimente US\$ 35 bilhões em 2025, divididos entre os setores onde a tecnologia tem grande potencial para crescer, principalmente: videogames, eventos ao vivo, entretenimento em vídeos, varejo, mercado imobiliário, educação, saúde, engenharia e militar. Nesse caso as indústrias de videogames, eventos ao vivo e entretenimento visual ocupam $60 \%$ do mercado, como pode ser observado na Figura 2. Além disso, ainda segundo Bellini et al. (2016), a RV e a RA tem o potencial não só de criar novos mercados, mas também de mudar, completamente, mercados existentes. 
Figura 2 - Divisão da Indústria de RV/RA em 2025

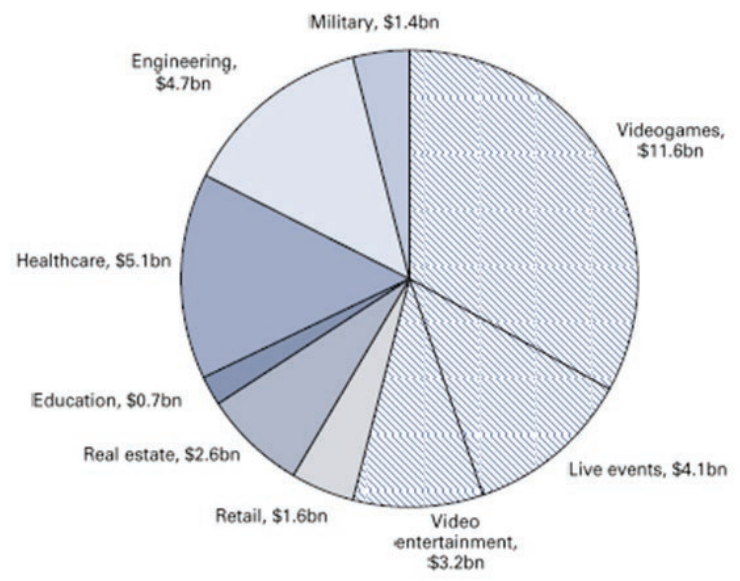

Fonte: Goldman Sachs Global Investment Research.

\section{Teoria Unificada de Aceitação e Uso da Tecno- logia}

A Teoria Unificada de Aceitação e Uso da Tecnologia (UTAUT) (VENKATESH et al., 2003) busca explicar a adoção da tecnologia, em que o indivíduo se sente confortável utilizando uma tecnologia que nunca teve contato. Para tanto, foi criado um modelo unificado, o qual integra oito estudos que trabalham com a aceitação do uso da tecnologia, são eles:

Theory of Reasoned Action (TRA): formulada por Fishbein e Ajzen (1975), essa teoria diz que a intenção do indivíduo é influenciada pelos sentimentos positivos ou negativos que ele possa ter, futuramente, pelo uso da tecnologia. Também leva em consideração uma norma subjetiva, que é a percepção do que as pessoas importantes para o indivíduo pensam do que ele deveria desempenhar em relação ao comportamento em questão.

Technology Acceptance Model (TAM): segundo Davis (1989), esse modelo tem como objetivo avaliar o comportamento de utilização da tecnologia, estudando as atitudes para o uso de um sistema de informação, com base na utilidade percebida pelo indivíduo e pela facilidade de utilização. Esse modelo evidencia que existem muitas restrições e competências que limitam a liberdade de utilização da tecnologia.

Motivational Model (MM): advindo da Teoria da Motivação, esse modelo estuda as motivações intrínsecas e extrínsecas que determinam o comportamento e levam ou não a adoção pelo indivíduo de novas tecnologias. Davis, Bagozzi e Warshaw (1992) utilizaram desse modelo
Theory of Planned Behavior (TPB): Teoria de Ajzen (1991), que estende a TRA adicionando o conceito de Controle Comportamental Percebido como determinante da intenção de uso da tecnologia. Tem como construtos principais a atitude para o comportamento, normas subjetivas e controle comportamental percebido.

Modelo Combinado TAM/TPB: Um modelo combinado entre a Theory of Planned Behavior e a Technology Acceptance Model, introduzido por Taylor e Todd (1995), que tem os seus principais construtos a atitude para o comportamento, normas subjetivas e controle comportamental percebido.

Model of PC Utilization: analisa a aceitação e o uso da tecnologia com base na complexidade, ajuste do trabalho, consequências de longo prazo, afeto ao uso, fatores sociais e condições facilitadoras. Thompson (1991) adaptou essa teoria para entender a aceitação e uso de computadores. Construtos utilizados: consequências de longo prazo, afeto ao uso, fatores sociais, condições facilitadoras, ajuste ao trabalho e complexidade.

Innovation Difusion Theory (IDT): Explica o processo de decisão da inovação e determinar os fatores que influenciam a taxa de adoção e as categorias adotadas (ROGERS, 1995). Moore e Benbasat (1996 apud VENKATESH et al., 2003) o adaptaram para que pudesse ser usado em estudos de adoção tecnológica individual. São construtos da teoria: vantagem relativa, facilidade de uso, demonstrativo de resultado, julgamento, visibilidade, imagem, compatibilidade e a voluntariedade.

Social Cognitive Theory (SCT): Baseia-se na ideia de que o comportamento do indivíduo é, simultaneamente, moldado por fatores internos, de personalidade e, também, pelo ambiente (BANDURA, 1986). São construtos da teoria: expectativas de resultado, autoeficácia, ansiedade e o efeito.

Com o objetivo de criar um modelo ainda mais completo, Vankatesh et al. (2003) desenvolveram a Teoria Unificada de Aceitação e Uso da Tecnologia (UTAUT). Para isso, realizaram uma comparação com todos esses modelos e fizeram estudos com quatro organizações que estavam introduzindo uma nova tecnologia. Essa teoria gerou um modelo integrado, que apresenta quatro conceitos determinantes da intenção do uso de tecnologia e quatro moderadores (Figura 3). 
Figura 3 - Modelo UTAUT

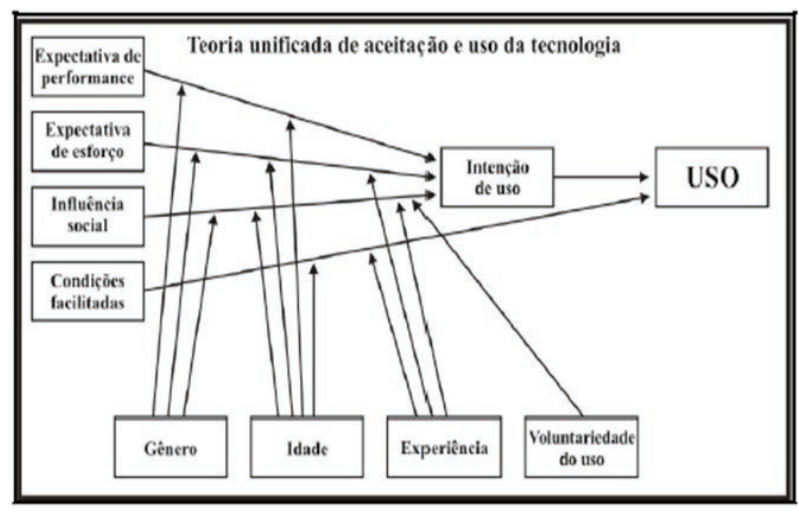

Fonte: (BOBSIN; VISENTINI; RECH, 2009, p. 102.)

Os conceitos determinantes são: a Expectativa de performance, que, resumidamente, é o grau que o indivíduo acredita que usando o sistema ele terá melhoria de desempenho no trabalho. A Expectativa de esforço, que se refere ao grau de facilidade relacionado ao uso do sistema. A Influência social é grau de percepção do indivíduo em relação aos demais quanto à crença destes para a necessidade de uma nova tecnologia ser usada ou não. E, por último, as Condições facilitadoras, que constituem o grau pelo qual se acredita que existe uma infraestrutura organizacional para comportar o uso do sistema.

E os construtos moderadores da intenção e o uso da tecnologia são: o gênero, a idade, a voluntariedade do uso e a experiência do indivíduo.

Assim, o UTAUT ajudou o avanço da pesquisa de aceitação e uso da tecnologia, unificando os modelos mais difundidos anteriormente e acoplando construtos moderadores. Os autores validaram o modelo por meio de estudos empíricos e chegaram à conclusão que este explica 70\% da variância da intenção de uso.

Dentre os estudos que utilizam o modelo UTAUT para análise de aceitação de tecnologias, é possível citar o estudo de Park, Yang e Lehto (2007) que procurou identificar características na adoção de tecnologias móveis por consumidores chineses, os de Almeida e Albertin (2014) que objetivou analisar a adoção e o uso da TI pelos consultores na Rede Social Corporativa e o de Leal et al. (2011), que buscou identificar fatores determinantes da aceitação do uso de tecnologias de informação na educação a distância, Todos esses estudos utilizaram modelos UTAUT adap- tados as situações e analisaram quantitativamente seus trabalhos.

\section{Método}

No estudo foi utilizada uma pesquisa quantitativa com caráter descritivo, em que foi utilizado o método survey para coleta de dados.

A pesquisa levantou informações sobre a aceitação dos possíveis usuários (clientes) frente ao uso da RA por meio de um aplicativo web. O método de obtenção dos dados da pesquisa foi pela aplicação de questionários a um grupo de pessoas participantes a fim de verificar a aceitação da tecnologia. Inicialmente, as pessoas foram convidadas a utilizar o aplicativo e, em seguida, respondiam um questionário.

Os questionários foram aplicados de dois modos. O primeiro, virtualmente, por meio da distribuição de links via e-mail ou mídias sociais para pessoas especificas. Nesse formato, os respondentes utilizavam o seu próprio computador para explorar o aplicativo e, em seguida, responder ao questionário. $\mathrm{O}$ segundo método foi realizado, presencialmente, em uma sala equipada com computador e webcam disponibilizada para a utilização do software e aplicação do questionário nos mesmos. O questionário aplicado foi uma adaptação do modelo UTAUT, proposto por Venkatesh et al. (2003).

No âmbito das aplicações de RA, testes utilizando o modelo UTAUT são pouco difundidos. Assim, com base de estudos do modelo em outros tipos de tecnologia e no modelo em si aplicado por Vankatesh et al. (2003) foi adaptado um questionário (Quadro 1) para aplicação com indivíduos que tiveram contato com algum aplicativo de RA que, para o nosso estudo, foi o Rayban Virtual Mirror (http://www.ray-ban.com/international/virtual-mirror). Esse aplicativo foi escolhido para o estudo, pois é, facilmente, acessado via internet, é parte de ação de marketing de uma empresa cuja marca tem presença mundial e uma boa experiência do usuário frente a RA. Como mencionado, o questionário foi estruturado com base no modelo formulado por Venkatesh et al. (2003) e é composto por 19 itens, que são apresentados no Quadro 1. Foi utilizada uma escala de avaliação Likert de 5 pontos, em que 1 representava "Discordo totalmente" e 5 "Concordo totalmente". 
Quadro 1 - Questionário adaptado do modelo UTAUT.

\begin{tabular}{|c|}
\hline Expectativa de Esforço \\
\hline 1A: Minha interação com o Ray-Ban Virtual Mirror foi clara e compreensível. \\
\hline 1B: 0 Ray-Ban Virtual Mirror foi fácil de se usar. \\
\hline 1C: Aprender a operar o Ray-Ban Virtual Mirror foi fácil para mim. \\
\hline $\begin{array}{l}\text { 1D: } 0 \text { uso do Ray-Ban Virtual Mirror é complicado, é difícil entender o que está } \\
\text { acontecendo. }\end{array}$ \\
\hline Expectativa de performance \\
\hline $\begin{array}{l}\text { 2A: } 0 \text { uso do Ray-Ban Virtual Mirror não terá nenhum efeito na minha escolha de } \\
\text { compra. }\end{array}$ \\
\hline $\begin{array}{l}\text { 2B: } 0 \text { uso do Ray-Ban Virtual Mirror pode diminuir o tempo necessário no processo de } \\
\text { compra. }\end{array}$ \\
\hline 2C: Eu me senti apreensivo usando o Ray-Ban Virtual Mirror. \\
\hline 2D: 0 Ray-Ban Virtual Mirror me intimidou um pouco. \\
\hline Intenção de uso \\
\hline 3A: Eu pretendo usar o Ray-Ban Virtual Mirror nas próximas compras. \\
\hline 3B: Eu prevejo que irei usar o Ray-Ban Virtual Mirror nas próximas compras. \\
\hline 3C: Eu planejo usar o Ray-Ban Virtual Mirror nas próximas compras. \\
\hline Influencia social \\
\hline $\begin{array}{l}\text { 4A: Pessoas que influenciam meu comportamento, pensam que eu deveria usar } 0 \\
\text { Ray-Ban Virtual Mirro. }\end{array}$ \\
\hline $\begin{array}{l}\text { 4B: Pessoas que são importantes para mim, pensam que eu deveria usar o Ray-Ban } \\
\text { Virtual Mirror. }\end{array}$ \\
\hline 4C: Usar o Ray-Ban Virtual Mirror me proporciona um status. \\
\hline Condições facilitadoras \\
\hline 5A: Eu tenho o controle do uso do Ray-Ban Virtual Mirror. \\
\hline 5B: Eu tenho o conhecimento para usar o Ray-Ban Virtual Mirror. \\
\hline 5C: 0 Ray-Ban Virtual Mirror não é compatível com outros sistemas que utilizo. \\
\hline 5D: Um guia foi disponibilizado para mim para uso do Ray-Ban Virtual Mirror. \\
\hline 5E: 0 uso do Ray-Ban Virtual Mirror é compatível com meu estilo de compra. \\
\hline
\end{tabular}

Fonte: do autor

Para provar a confiabilidade do questionário, foi calculado o Alfa de Cronbach, que mede a correlação entre respostas em um questionário por meio da análise do perfil das respostas dadas pelos respondentes como uma forma de estimar a confiabilidade de um questionário aplicado a uma pesquisa. Trata-se de uma correlação média entre as perguntas. Esse coeficiente varia entre $0 \mathrm{e}$ 1, quanto mais próximo do valor máximo, maior a confiabilidade das escalas (MALHOTRA, 2006; HAIR et al, 2005). Como é possível ser observado na Tabela 1 , o alfa dos fatores variou entre 0,909 e 0,641. O limite mínimo aceito para o alfa é de 0,60, o que aponta uma boa confiabilidade da escala do questionário. Não foi possível calcular o alfa de Cronbach do fator seis (6) por conter apenas um item. Porém, este representa 5,46\% da variância explicada, não influenciando na confiabilidade da pesquisa.

\footnotetext{
Por fim, como é possível observar na Tabela 2,
}

foram encontradas as estatísticas descritivas da amostra, contendo a média e desvio padrão das perguntas feitas no questionário.

Analisando esses dados descritivos é possível concluir que o construto Expectativa de Esforço, composto das questões 1A, 1B, 1C e 1D (Quadro 1) tem as maiores médias nas suas questões, o que indica as qualidades da ferramenta, pois seu uso pelos respondentes do questionário foi compreensível, fácil de se usar e de aprender a operar se necessário. É possível, também, destacar, entre as médias dos itens, as do construto Intenção de Uso, que manteve média em todos os itens perto de três e com alto desvio padrão entre as respostas, o que indica que as respostas nesses itens variaram bastante.

Tabela 2 - Estatísticas Descritivas da Amostra.

\begin{tabular}{|c|c|c|}
\hline Item & Média & $\begin{array}{l}\text { Desvio } \\
\text { Padrão }\end{array}$ \\
\hline $\begin{array}{l}\text { 1A: Minha interação com o Ray-Ban Virtual Mirror foi clara e } \\
\text { compreensível. }\end{array}$ & 4,07 & 0,87 \\
\hline 1B: 0 Ray-Ban Virtual Mirror foi fácil de se usar. & 3,95 & 0,91 \\
\hline 1C: Aprender a operar o Ray-Ban Virtual Mirror foi fácil para mim. & 4,07 & 0,81 \\
\hline $\begin{array}{l}\text { 1D: } 0 \text { uso do Ray-Ban Virtual Mirror é complicado, é difícil enten- } \\
\text { der } 0 \text { que está acontecendo. }\end{array}$ & 1,85 & 0,86 \\
\hline $\begin{array}{l}\text { 2A: } 0 \text { uso do Ray-Ban Virtual Mirror não terá nenhum efeito na } \\
\text { minha escolha de compra. }\end{array}$ & 2,36 & 1,11 \\
\hline $\begin{array}{l}\text { 2B: } 0 \text { uso do Ray-Ban Virtual Mirror pode diminuir o tempo } \\
\text { necessário no processo de compra. }\end{array}$ & 3,65 & 1,12 \\
\hline 2C: Eu me senti apreensivo usando o Ray-Ban Virtual Mirror. & 2,01 & 1,06 \\
\hline 2D: 0 Ray-Ban Virtual Mirror me intimidou um pouco. & 1,68 & 0,84 \\
\hline $\begin{array}{l}\text { 3A: Eu pretendo usar o Ray-Ban Virtual Mirror nas próximas } \\
\text { compras. }\end{array}$ & 3,26 & 1,17 \\
\hline $\begin{array}{l}\text { 3B: Eu prevejo que irei usar o Ray-Ban Virtual Mirror nas próximas } \\
\text { compras. }\end{array}$ & 3,14 & 1,21 \\
\hline 3C: Eu planejo usar o Ray-Ban Virtual Mirror nas próximas compras. & 3,11 & 1,19 \\
\hline
\end{tabular}




\begin{tabular}{|c|c|c|}
\hline $\begin{array}{l}\text { 4A: Pessoas que influenciam meu comportamento, pensam que eu } \\
\text { deveria usar o Ray-Ban Virtual Mirro. }\end{array}$ & 2,38 & 1,03 \\
\hline $\begin{array}{l}\text { 4B: Pessoas que são importantes para mim, pensam que eu } \\
\text { deveria usar o Ray-Ban Virtual Mirror. }\end{array}$ & 2,35 & 0,99 \\
\hline 4C: Usar o Ray-Ban Virtual Mirror me proporciona um status. & 2,06 & 1,04 \\
\hline 5A: Eu tenho o controle do uso do Ray-Ban Virtual Mirror. & 3,70 & 1,17 \\
\hline 5B: Eu tenho o conhecimento para usar o Ray-Ban Virtual Mirror. & 3,90 & 0,92 \\
\hline $\begin{array}{l}\text { 5C: } 0 \text { Ray-Ban Virtual Mirror não é compatível com outros sistemas } \\
\text { que utilizo. }\end{array}$ & 1,93 & 1,08 \\
\hline $\begin{array}{l}\text { 5D: Um guia foi disponibilizado para mim para uso do Ray-Ban } \\
\text { Virtual Mirror. }\end{array}$ & 2,32 & 1,25 \\
\hline $\begin{array}{l}\text { 5E: } 0 \text { uso do Ray-Ban Virtual Mirror é compatível com meu estilo } \\
\text { de compra. }\end{array}$ & 3,10 & 1,16 \\
\hline
\end{tabular}

Fonte: do autor

\subsection{Fatores determinantes}

Cada fator encontrado na análise fatorial (Tabela 1) foi classificado e interpretado com base nos estudos da Teoria Unificada de Aceitação e Uso da Tecnologia, onde cada item do questionário estava incluído dentro de um dos cinco construtos descritos por Vankatesh et al. (2003). Assim, foi possível distribuir seis fatores dentre os cinco construtos, sendo eles: Expectativa de Performance, Expectativa de Esforço, Influência Social, Condições Facilitadoras e Intenção de Uso. Na análise fatorial, foram descobertos seis fatores, porém, não será analisado o fator seis, separadamente, por explicar, apenas, $5,46 \%$ da variância dos dados e por conter, apenas, um item.

$O$ primeiro fator que tem a maior variância explicada de 23,63\%, é composto pelos itens 3A, 3B, 3C e 5E. Assim, em sua maioria é formado por itens advindos do construto Intensão de Uso, que indica que este construto contém os principais determinantes da aceitação do uso da Realidade Aumentada no aplicativo Rayban Virtual Mirror. A média dos itens que compõem este fator se manteve um pouco acima de três, sugerindo uma leve tendência ao uso do Rayban Virtual Mirror futuramente.

$\mathrm{O}$ segundo fator pode ser classificado no contexto da Expectativa de Esforço, pois é formado, apenas, pelos itens desse construto, 1A, 1B e 1C. Esse fator apresenta o quanto foi fácil para os usuários explorarem a ferramenta de RA. Como mencionado por Davis (1989), haverá maior aceitação de certa tecnologia quanto maior for a facilidade de uso percebida. Nesse fator, foi possível identificar $18,93 \%$ da variação do uso na pesquisa, apresentando fatores importantes para a aceitação do uso da tecnologia pesquisada. A média encontrada desses três itens, também, foi alta, como pode ser visto na Tabela 2, o que sugere que os usuários do aplicativo tiveram facilidade em utilizar a ferramenta.

O terceiro fator é composto por itens de três dos construtos identificados por Venkatesh et al. (2003): os itens 1D, 2C e 2D e 5C, sendo ele Expectativa de Esforço (item 1D), Expectativa de Performance (itens 2C e 2D) e Condições Facilitadoras (item 5C), que explica 10,26\% da intenção de uso dos participantes. Como dois dos quatro fatores são do construto Expectativa de Performance, que reflete o quanto a ferramenta aumentará a qualidade do serviço oferecido, é possível concluir que este construto é importante para a aceitação do uso da RA. É possível, ainda, reforçar a importância desse construto, pois o sexto fator encontrado é formado, apenas, pelo item $2 \mathrm{~B}$, que explica 5,46\% da variância da aceitação do uso.

O quarto fator representa o construto Influência Social, uma vez que é formado em sua totalidade pelos itens $4 \mathrm{~A}, 4 \mathrm{~B}$ e 4C. No modelo proposto por Venkatesh et al. (2003), a influência social trata da percepção do usuário sobre a influência que demais membros podem ter em sua convivência social na utilização do sistema. Esse fator explica $7,9 \%$ da aceitação e uso da RA. Em se tratando do Rayban Virtual Mirror, a média dos itens desse fator ficaram abaixo de dois, mostrando que os usuários não percebem uma influência social na utilização do sistema.

Por último, o quinto fator explica $6,38 \%$ da variância dos dados e é formado pelos itens $5 \mathrm{~A}$ e $5 \mathrm{~B}$, ambos do construto Condições Facilitadoras, que reflete o quanto os respondentes acham que as condições da ferramenta facilitam o uso da RA.

Assim, com a análise fatorial, foi possível associar os fatores que mais explicam a variação dos dados da pesquisa aos construtos destacados no modelo UTAUT por Venkatesh et al. (2003) que explicam a aceitação e uso de tecnologias. Dentre estes construtos, a Intenção de Uso tem o maior autovalor, e, por consequência, explica a maior variação da aceitação da RA como ferramenta de marketing 
pelos usuários, seguidamente, a Expectativa de Esforço, também, explica uma grande parcela da variação na aceitação da tecnologia, o que nos dá diretrizes para o desenvolvimento de ferramentas de marketing que utilizem RA.

\section{Conclusões}

O estudo fez uma análise de fatores importantes na aceitação e uso da tecnologia de RA como uma ferramenta de Marketing, por usuários do aplicativo Rayban Virtual Mirror, sob a ótica da UTAUT.

Com aplicação de uma adaptação do questionário da UTAUT, foi possível confirmar os construtos descobertos por Venkatesh et al. (2003) como determinantes na aceitação de uso, Expectativa de Performance, Expectativa de Esforço, Intenção de Uso, Influência Social e Condições Facilitadoras.

Dentre esses construtos evidenciados no estudo de Venkatesh et al. (2003), dois se destacam na aceitação e uso da RA como estratégia de Marketing para a amostra: a Intenção de Uso que reflete a intenção dos usuários de utilizar a ferramenta futuramente, assim como fazer proveito da RA e a Expectativa de Esforço, que confirma que quanto maior a facilidade no uso da tecnologia maior será a aceitação da mesma.

Como contribuição da pesquisa, ficam direcionadas estratégias que possam ser adotadas para a melhor utilização da RA no marketing. Recomenda-se que as empresas que pretendem utilizar esse tipo de tecnologia deem atenção no que se trata do desenvolvimento de uma ferramenta que facilite o processo de compra de produtos, que faça com que o usuário repita o processo futuramente e procure sempre utilizar da ferramenta, assim como atenção para o desenvolvimento de um software que seja fácil e intuitivo para o usuário. Não pode, também, desconsiderar a atenção para os outros três construtos descritos anteriormente, Expectativa de Performance, Influência Social e Condições Facilitadoras.

Com os resultados, apesar de suas limitações, resultantes da especificação da amostra, por se tratar de uma tecnologia nova e pouco difundida, e de existirem poucos estudos na área, espera-se auxiliar e orientar gestores no uso de a RA como estratégia de Marketing.

Espera-se, também, que este estudo contribua tanto para pesquisas futuras relacionadas à aplicação da Realidade Aumentada no cenário de negócios quanto para pesquisas que utilizem o método UTAUT para análise de novas tecnologias.

\section{Referências}

AJZEN, I.; FISHBEIN, M. Belief, attitude, intention and behavior: an introduction to theory and research, 1975.

AJZEN, I. The theory of planned behavior. Organizational Behavior and Human Decision Processes, p. 179-211, Dez. 1991.

AZUMA, R. A survey of augmented reality. Presence: Teleoperators and Virtual Environments, v .6, n. 4, p. 355385, Aug. 1997.

AZUMA, R. et al. Recent advances in augmented reality. IEEE Computer Graphics and Applications, v. 21, n. 6, p. 34-47, 2001.

ALMEIDA, R.; ALBERTIN, A. L. Análise dos fatores determinantes à adoção de rede social corporativa com aplicação do modelo. Revista Eletrônica Gestão e Serviços, v. 5, n. 2, p. 994-1013, 2014.

BANDURA, A. Social foundations of thought and action: a cognitive social theory. Englewood Cliffs, New York: Pretince Hall, 1986.

BELLINI, H et al. Virtual \& augmented reality: understanding the race for the next computing platform. The Goldman Sachs Group, Inc. 2016. Disponível em: <http:// www.goldmansachs.com/our-thinking/pages/technology-driving-innovation-folder/virtual-and-augmented-reality/report.pdf>.

BOBSIN, D.; VISENTINI, M. S.; RECH, I. Em busca do estado da arte do UTAUT: ampliando as considerações sobre o uso da tecnologia. RAI: revista de Administração e Inovação, v. 6, n. 2, p. 99-118, 2009.

CATTELL, R. B. The scree test for the number of factors. Multivariate Behavioral Research, v. 1, n. 2, p. 245-276, 1966.

CARDOZO, D. Realidade aumentada nas áreas de engenharia, arquitetura e construção. 2015. Disponível em: <http://danielcardozo.com.br/2015/03/26/artigo-realidade-aumentada-areas-engenharia-arquitetura-construcao/>. Acesso em: 25 jul. 2015.

CASTANHA, L. A. Como a realidade aumentada pode ajudar nos treinamentos as empresas. 2015. Disponível em: <http://corporate.canaltech.com.br/noticia/nego- 
cios/como-a-realidade-aumentada-pode-ajudar-nos-treinamentos-as-empresas-45359/>. Acesso em: 25 jul. 2015.

DAVIS, F. D. Perceived usefulness, perceived ease of use, and user acceptance of information technology. MIS quarterly, p. 319-340, 1989.

DAVIS, F. D.; BAGOZZI, R. P.; WARSHAW, P. R. Extrinsic and intrinsic motivation to use computers in the workplace1. Journal of Applied Social Psychology, v. 22, n. 14, p. 1111-1132, 1992.

GRANDELLE, R.; JANSEN, T. Com realidade aumentada e visitas remotas, museus têm novas formas de explor acervos. 2015. Disponível em: <http://oglobo.globo. $\mathrm{com} /$ sociedade/historia/com-realidade-aumentada-visitas-remotas-museus-tem-novas-formas-de-expor-acervos-16241386>. Acesso em: 9 jul. 2015.

HAIR, J. F. et al. Análise multivariada de dados. Porto Alegre: Bookman, 2009.

INSLEY, S. Augmented reality: merging the virtual and the real. Oregon: Oregon State University, 2003.

KIRNER, C.; SISCOUTTO, R. Realidade virtual e aumentada: conceitos, projetos e aplicações. Livro do Pré-Simpósio, Petrópolis - RJ. 2007. Disponível em: $<$ http://www.realidadevirtual.com.br>. Acesso em: 16 abr. 2014.

LATTIN, J.; CARROLL, J. D.; GREEN, P. E. Análise de dados multivariados. São Paulo: Cengage Learning, 2011.

MALHOTRA, N. K. Pesquisa de marketing: uma orientação aplicada. Porto Alegre: Bookman, 2006.

MILGRAM, P.; KISHINO, F. A taxonomy of mixed reality visual displays, IEICE (Institute of Electronics, Information and Communication Engineers) Transactions on Information and Systems, Special issue on Networked Reality, dec. 1994.

MILGRAN, P. et al. Augmented reality: a class of displays on the reality-virtuality continuum. Telemanipulator and Telepresence Technologies, v. 2351, p. 282-292. 1994.

MORIE, J. F. Inspiring the future: merging mass communication, art, entertainment and virtual environments. Computer Graphics, v. 28, n. 2, p. 135-138, 1994.
LEAL, E. A. et al. Utilização da análise fatorial para identificação dos fatores determinantes da aceitação do uso de tecnologias de informação na educação a distância, 2011.

PARK, J.; YANG, S.; LEHTO, X. Adoption of mobile technologies for chinese consumers. Journal of Electronic Commerce Research, v. 8, n. 3, p. 196-206, 2007.

RODELLO, I. A.; BREGA, J. R. F. Realidade virtual e aumentada em ações de marketing. Realidade virtual e aumentada: aplicações e tendências, p. 45, 2011.

RODELLO, I. A. et al. Realidade misturada: conceitos, ferramentas e aplicações. Revista Brasileira de Computação Aplicada, v. 2, n. 2, p. 2-16, 2010.

ROGERS, E. M. Diffusion of innovation. The Free Press, 1995.

SUNDARAVEJ, T. Empirical validation of unified theory of acceptance and use of technology model. Journal of Global Information Technology Management, v. 13, n. 1, p. 5-27, 2010.

SUTHERLAND, I. E. A head-mounted three dimensional display. In Proceedings of the December 9-11, 1968, fall joint computer conference, part I (AFIPS ‘68 (Fall, part I)). ACM, New York, USA, p. 757-764, 1968.

TAYLOR, S.; TODD, P. A. Understanding information technology usage: a test of competing models. Information Systems Research, v. 6, n. 2, p. 144-176, 1995.

THOMPSON, R. L.; HIGGINS, C. A.; HOWELL, J. M. Personal computing: toward a conceptual model of utilization. MIS Quarterly, p. 125-143, 1991.

VAN KLEEF, N.; NOLTES, J.; VAN DER SPOEL, S. Success factors for augmented reality business models. Study Tour Pixel, p. 1-36, 2010.

VENKATESH, V. et al. User acceptance of information technology: toward a unified view. MIS Quarterly, p. 425478, 2003.

WHITE, J.; SCHMIDT, D. C.; GOLPARVAR-FARD, M. Applications of augmented reality. Proceedings of the IEEE, v.102, n. 2, Feb. 2014. 